\title{
Review of Individual Tacit Knowledge Measurement
}

\author{
Zeng Zhi, Shen Junlong, Chen Xingzi, Xiang Gaoyue \\ Nanjing University of Chinese Medicine, College of Economics and Management, Nanjing, China
}

Email address:

zengzhinju@163.com (Zeng Zhi)

\section{To cite this article:}

Zeng Zhi, Shen Junlong, Chen Xingzi, Xiang Gaoyue. Review of Individual Tacit Knowledge Measurement. Humanities and Social Sciences. Vol. 4, No. 6, 2016, pp. 135-139. doi: 10.11648/j.hss.20160406.11

Received: August 23, 2016; Accepted: September 7, 2016; Published: October 13, 2016

\begin{abstract}
People have recognized that the vast majority of the human beings' knowledge (accounting for $90 \%$ of the total amount of knowledge) is tacit knowledge that it is difficult to express, difficult to code, in addition to the explicit knowledge which can be expressed and encoded, since Polanyi proposed and constructed "tacit knowledge" theory. As a result, the measurement of tacit knowledge has become one of the key objects in the research of knowledge management. The related achievement from different countries' scholars during the past 30 years will become an important asset for further research in the field, since Sternberg R.J., a famous American psychologist, initially discussed the measurement of tacit knowledge in 1987.
\end{abstract}

Keywords: Tacit Knowledge, Measurement, Methods, Practice

\section{Introduction}

A number of scholars from different fields such as philosophy, psychology, education, sociology, management science and computer science etc, started to do deeper research about tacit knowledge, since Michael Polanyi (1891-1976) has proposed and constructed the theory of "tacit knowledge" in his famous book "personal knowledge". Nowadays, scholars agree that tacit knowledge exists in our study, work and life. As the famous Austrian psychoanalyst, Sigmund Freud's sub-consciousness theory put forward the study of human consciousness extended to all human behavior and S. Freud became the most depth psychologist on human psychology, Polanyi's theory about tacit knowledge put forward the study of human knowledge expanded to a broader situation and Polanyi became the most depth of scholars on human knowledge. Since then, all human actions have been considered to Knowledgeable.

This is the new economy era featured with human knowledge's production, exchange, distribution and consumption .Knowledge has become the main resource and tool that can promote individual or organization to make money and improve core competence. In this situation, the explicit knowledge that can be perceived (visible and touchable), expressed, transferred and saved easily plays an important role and it has become an important resource that individual or organizations want to gain .At the same time, the tacit knowledge that was stored in people's own brain and can't explain explicitly has become the core competence of individual or organization. Management, education, psychology and information science attach great importance to measurement and analysis of explicit knowledge and have achieved plentiful research results for a long time. In recent years, along with the rise of knowledge management, knowledge economy has become the dominant economy in the 21 st century; measurement of tacit knowledge has become the central issue and one of the important subjects to be solved urgently in the research field of knowledge management.

In order to investigate the development of research on "tacit knowledge", this study take two ways to retrieve academic literature from the Steven database (EBSCOhost) : (1) With "Tacit Knowledge" as the title (TI) to retrieve , there were 1560 papers in the database (including journals, books, research reports, conference papers); (2) With "Tacit Knowledge" as the Subject words (SU) for retrieval, there were 3010 papers in the database (including journals, books, research reports, conference papers). The earliest journal articles with "tacit knowledge" as title was written published in "College English" (April 1969, Vol. 30), and the title is "Child as story teller: teaching literary concepts through tacit knowledge". This paper mainly discusses the guidance of tacit knowledge to teaching from the perspective of educational science. For more intuitive perceiving to the origin and development of the research on "tacit knowledge" in the 
western academic field, the study use "Tacit Knowledge" as the subject word (SU) in the "Steven database (EBSCOhost)" to carry out the literature search, and especially in-depth analysis the literature of the past 20 years (1996-2015).In the data analysis, this study discussed the development trend of the research on tacit knowledge with the number of academic literatures as an indicator (Figure 1).

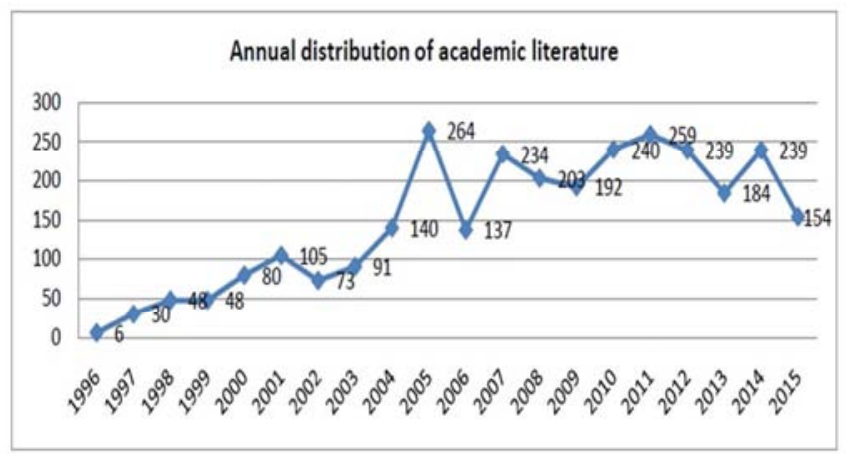

Figure 1. Annual distribution of academic literature of research on tacit knowledge.

In all of these documents, the problem about tacit knowledge measurement was an everlasting topic. Scholars were carried out a variety of research from different perspectives, different industries, and different disciplines.

\section{Discussion on the Feasibility of the Measurement of Tacit Knowledge}

From the perspective of surveying (especially psychometrics), whether a thing can be evaluated or measured primarily based on two aspects: namely, whether there is some variety (difference) or whether it can be quantified (testability).

\subsection{The Analysis on the Differences of Tacit Knowledge}

As we all know, things in the world are vastly different, even if there are individual differences in the same kind of things, and there don't exist two identical things in the world. Just as in our daily lives, some people are fat and some are thin; some are handsome and some are ugly. The difference between people is more than the external differences. As early as 2,500 years ago, in ancient China the great educator and thinker Confucius has assessed them by his own observations of the individual differences in his disciples, and he has adopted a classification mode, in accordance with the superior, medium and inferior, divided into nine types of people. Tacit knowledge, as a mental capacity or psychological characteristics of the individual, the differences is bound to exist. Even people who get the same skills, the tacit knowledge they possess is different. For example, the acrobat or the kung fu masters who exhibit superb skill in their field but different acrobats or kung fu masters' tacit knowledge may be a big difference. Just because there are individual differences in tacit knowledge, it is necessary to conduct a scientific assessment on it.

\subsection{The Tacit Knowledge of Testability Analysis}

Mencius once said, "Weigh a scale, to know the weight of the things; the amount of a measure, to know the length of the things. What is the case, the human heart is so." ( From "Mencius . King Hui of Liang") There are two important saying in the history of scientific psychological measurement. One is the famous psychologist E. L. Thorndike said, "Whatever exists at all exists in some amount. To know it thoroughly involves knowing its quantity as well as its quality" [2]; another is the famous Education survey scientist W.A McCal said: "Anything that exists in amount can be measured" [3]. With the development of science and technology, people can not only make more accurate measurements about the length, weight, temperature and other physical properties , but we also can keep trying to use various methods to measure psychological characteristics (such as thinking, mood, temperament, personality, etc.).

Accordingly, since tacit knowledge is an objective reality and is relatively stable for the individual, it is possible for us to carry on quantitative analysis by some special measuring methods. Of course, tacit knowledge has a subtle characteristic; its measurement cannot be as easy as the measurement of length, mass, temperature, size and other physical characteristics of the object. We cannot measure it directly; we can only speculate the level and characteristics of the individuals' tacit knowledge through some special methods.

In short, although it is difficult to make tacit knowledge clear and tacit knowledge is highly individualized, the measurement is always much difficult than the measurements of physics, but a lot of studies have shown that tacit knowledge can be effectively measured.

\section{Method and Practice of Tacit Knowledge Measurement}

\subsection{Study about the Evaluation Method of Tacit Knowledge Measurement}

As described above, since the individual tacit knowledge has characteristics of difficult to express, highly individualized, cultural dependent, unconsciousness and so on .It is bound to bring a lot of difficulties if we make the evaluation directly to tacit knowledge which is hard to imitate, express and spread. The problems like how to obtain, define, capture and quantify the tacit knowledge have become obstacles during the study . But it cannot be the reason to prevent us from exploring and measuring tacit knowledge. Tacit knowledge has lots of special characteristics, but it is attached to specific cognitive subjects and has great affects on the activity and practice of cognitive subjects. Therefore, the measurement of tacit knowledge can be implied in an indirect way, the measurement can be started from the study of the extravert behavior and cognitive characteristics that tacit 
knowledge shows; it is easier to measure the individual tacit knowledge by analyzing cognitive subject's way of thinking and the characteristics of their behavior to quantify. [4]

Tacit knowledge is difficult to be measured and evaluated directly, according to different objects and purposes, scholars around the world did many assessment studies on tacit knowledge by evaluating individuals' behavior when they use the tacit knowledge (experience or intuition) to cognize objective world and deal with the practical problems. And it has achieved great success. The methods can be divided into two categories: one is to develop a tacit knowledge scale aiming at the target groups, using methods like situational judgment test to get more comprehensive measurement, analysis and assessment about tacit knowledge of target groups; Another method is based on the fuzzy characteristics of tacit knowledge, from the perspective of construct validity, a corresponding measurement index system is set up by deeply understanding the crowd's tacit knowledge in certain areas or sectors, and then tacit knowledge is able to be measured by applying the fuzzy comprehensive measurement method or multi-index comprehensive measurement method braised on language information. [5] The first kind of method starts from the structure of tacit knowledge, by understanding and grasping a particular situation, a series of problem-solving questions coming from particular situation at work and the corresponding number of the methods are developed, and then we can use situational judge measurement of tacit knowledge to accomplish a comprehensive measurement aiming at the target group. These methods come from real situations, so the reliability and validity of measurements are good, but the process is complicated. The second category is also from the structure, but what different from the first kind of method is that the theoretical basis originates in ambiguous concept of tacit knowledge, after deeply understanding the crowd's tacit knowledge in certain areas or sectors, a new-different measurement index system is established, then the new system can be used in conducting a comprehensive measurement. Such methods of quantitative and qualitative analysis have a very good combination, and the measurement process is relatively simple, the results are scientific and objective. Specifically, in current, the main methods for the evaluation of tacit knowledge are Psychological Measurement, Simulation
Method of Real-World Performance, Trait and Behavioral Check List, Critical Incident, Thematic Apperception Test, Assessment Center and Difference between Expertise and Novice. [6]

\subsection{Research on the Practice of Tacit Knowledge Measurement}

Some theories were found during sorting documents concerning measurement study of tacit knowledge, the famous psychologist Robert J. Sternberg (1987) was the scholar who the first proposed that tacit knowledge can be measured and carried out measurement practice formally. He and Richard K. Wangner presented that tacit knowledge can be divided into three types: self-management, managing others and managing tasks. Through empirical research, and developed tacit knowledge inventory for managers (TKIM) [7].

Since then, foreign scholars began to focus on the basis of Sternberg's tacit knowledge assessment research, starting from different angles and different areas, and achieved great success in certain areas. For example: Hedlund J, Forsythe GB \& Horvath J A. developed Tacit Knowledge Scale For Military Leadership (MLTKS) [8]; Nancy Leonard \& Gary S.Insch (2005) developed Tacit Knowledge Scale Academia (ATKS) [9]; Peter Busch (2008) developed Information Systems Staff's Tacit Knowledge Scale (ISSTKS) [10]; By learning from their mentor Sternberg's the Triarchic Theory of Human Intelligence in Beyond IQ, Cianciolo, A.T \& Grigorenko, E. L (2006) designed Practical Ability Tacit Knowledge Investigation (PATKI) centering three typical situation in triarchic theory. [11] These scales are empirically analyzed in the form of self-report which is included in Likert scale. It has received good results when doing tacit knowledge measurement for expertise in specific areas. In recent years, based on the concept definition of tacit knowledge, Ellis, R. (2008) used the three test tools (Methods) on tacit knowledge of measurement and analysis, namely: oral narrative test, timed grammatical judgment test, and Elicited imitation test. [12] The use of these testing methods (tools) is to improve the reliability and validity of the evaluation results of tacit knowledge. [13]

Chart 1. Introduction of representative measurement tools.

\begin{tabular}{|c|c|c|c|}
\hline Researchers or research team & Subject areas & Object of measurement & Measuring tool \\
\hline R.Wangner \& R.Sternberg & Psychology\& Management & General manager & Tacit Knowledge Inventory for Managers \\
\hline $\begin{array}{l}\text { Hedlund J., Forsythe G. \& } \\
\text { Horvath J. }\end{array}$ & Psychology & Military Leader & $\begin{array}{l}\text { Tacit Knowledge Scale For Military } \\
\text { Leadership }\end{array}$ \\
\hline N. Leonard \& G. S.Insch & Management \& Education & Academic staff in Universities & Tacit Knowledge Scale Academia \\
\hline Peter Busch & $\begin{array}{l}\text { Management \&Information } \\
\text { Science }\end{array}$ & Information Systems Staff & $\begin{array}{l}\text { Information Systems Staff's Tacit } \\
\text { Knowledge Scale }\end{array}$ \\
\hline TANG Kexin & Psychology & Knowledge worker & $\begin{array}{l}\text { Tacit Knowledge Inventory For Managers } \\
\text { (TKIM-R) }\end{array}$ \\
\hline LI Zuoxue & Management & $\begin{array}{l}\text { Enterprise staff, University } \\
\text { Graduate }\end{array}$ & $\begin{array}{l}\text { Individual Tacit Knowledge Ability } \\
\text { Questionnaire }\end{array}$ \\
\hline XM Liu, JC Xiao, XW Zhao & Management & General staff & Scale of Individual Tacit Knowledge \\
\hline
\end{tabular}

Chinese scholars' empirical research on tacit knowledge assessment has three main orientations: one was localizing mature measurement scales from the foreign scholars and used the new inventory to measure certain industry groups. such as: 
Tang Kexin (2004) revised Richard K. Wangner \& Robert J. Sternberg's (1987) tacit knowledge inventory for managers (TKIM), evaluated and analyzed 254 knowledge workers. [14]; another was aiming to conduct tacit knowledge measurement in specific area, such as Wenjiao Yang, Zhijin Zhou analyzed the tacit knowledge in scientific research on the graduate students in six universities (2011) [15], Xiaokun Wang's tacit knowledge study is amied at insurance officer (2009) [16]; and the other was to rebuild the index system of the tacit knowledge for individual and make a new scale to carry out the measurement, such as Zuoxue Li (2008) reconstructed dimensions and measurement system concerning tacit knowledge for individual by summarizing researches and interviews from scholars around the world and the applied it into study [17]; Based on the existing research, Yong Zhou Li and Jing Peng (2012) designed individual tacit knowledge measurement index system targeting the research and development in enterprise, and selected the appropriate measurement methods for the associated characteristics among the different indicators and then implemented measurement and analysis [18]; With more than 20 organizations, 245 employees from Sichuan, Fujian, Jilin and SEM method is used to test validity of the formal one. A simple and effective scale of individual tacit knowledge has been proposed (Xuemei Liu, 2013). [19].

\section{Conclusions}

Through gathering, organizing and analyzing the documents about tacit knowledge from different countries, we found that although researches about tacit knowledge have been carried out in different fields especially in psychology and management, there has not formed a set of measuring tacit knowledge standard that is acceptable, simple and easy for different fields yet. The existing research on individual tacit knowledge measurement was limited to a particular industry or class. A general level of measurement development needs large sample data and tools innovated. Of course, the phenomenon also demonstrated the complexity of the tacit knowledge. Especially in China, researches were almost simple imitation and exploration. Actually, tacit knowledge has obvious cultural attachment, so there are differences between east and west countries. For example, the way of thinking under Chinese tradition culture would have some special points in the formation of tacit knowledge and index system. According to the influence of mode of thinking under the background of Chinese traditional culture on the tacit knowledge and the intuitive experience special cognitive mechanism, Q. Wang and other scholars found in some researches (2005) that individual tacit knowledge evaluation index can be defined as five aspects: the depth and breadth of knowledge, the ability to use imagery, the ability to Qu Xiang Bi Lei, and the ability to run through knowledge, emotion and meaning, the relative performance of tacit knowledge. [20]. In China, these results were only theoretical ideas, and the measurement of tacit knowledge was still in the exploratory stage, and needs to be strengthened and developed in the future.

\section{Acknowledgements}

This paper is one of the research results of "National Social Science Foundation of China (Project Number: 15BGL158)".

\section{References}

[1] Nigel Pleasants. Nothing is Concealed: De-centring Tacit Knowledge and Rules from Social Theory [J]. Journal for the Theory of Social Behavior, 1996, 26(3):233-255.

[2] Edward L. Thorndike. The Seventeenth Yearbook of The National Society For Study of Education (Part II) [M]. Bloomington: Public School Publishing Co. p., 1918:16.

[3] William A. McCall. Measurement [M]. New York: The Macmillan Company, 1939:15.

[4] ZOU Yajing. Evaluation Study of Corporate Personal Tacit Knowledge [D]. Changchun: Northeast Normal University, 2013:15-16.

[5] GUO Jian, SHEN Juntao. A Research on Individual's Tacit Knowledge Measurement [J]. SCIENTIFIC DECISION-MAKING, 2010(5):87-94.

[6] MENG Bin. The Research on Tacit Knowledge Structure and Its Inventory for T teachers' Teaching Practice [D]. Changchun: Jilin University, 2007:16.

[7] R.K. Wagner, R.J. Sternberg. Tacit Knowledge In Managerial Success [J].Journal of Business \& Psychology, 1987, 1(4): 301-312.

[8] Hedlund J, Forsythe G B, Horvath J A. Identifying and Assessing Tacit Knowledge: Understanding the Practical Intelligence of Military Leaders [J]. The Leadership Quarterly, 2003, 14(2), 117-140.

[9] Leonard Nancy, Insch Gary S. Tacit knowledge in academia: a proposed model and measurement scale [J]. The Journal of Psychology, 2005, 139(6): 495-512.

[10] Peter Busch. Tacit Knowledge in Organizational Learning [M]. New York: IGI Publishing, 2008: 424-449.

[11] Cianciolo, A. T., Grigorenko, E. L., Jarvin, L., Gil, G., Drebot, M. E., \& Sternberg, R. J. Practical intelligence and tacit knowledge: Advancements in the measures of developing expertise [J]. Learning and Individual Differences, 2006(16): 235-253.

[12] TL Cox, J Bown, J Burdis. Exploring Proficiency-Based vs. Performance-Based Items With Elicited Imitation Assessment [J].Foreign Language Annals, 2015, 48(3): 350-371.

[13] YU Hong. On Measuring L2 Learners' Implicit Knowledge with Elicited Imitation Tests [J]. Journal of Mianyang Normal University, 2015, 34(4): 42-46.

[14] TANG Kexin. The Revision of Tacit Knowledge Inventory For Managers [D]. Chongqing: Southwest China Normal University, 2004: 23-28.

[15] YANG Wenjiao, ZHOU Zhijin. An Empirical Study on Tacit Knowledge of Graduate Students -- Based on The Questionnaire Survey of Six Universities [J]. Higher Education Exploration, 2011(6): 61-66. 
[16] WANG Xiaokun. Study On The Job Tacit Knowledge Structure of Salespersons In Insurance [D]. Shenyang: Shenyang Normal University, 2009:45-50.

[17] LI Zuoxue. Evaluation and Management of Tacit Knowledge [M]. Dalian: Dalian University of Technology Press, 2008:107-130.

[18] LI Yongzhou, PENG Jing. Measure of Individual Tacit Knowledge and its Application in R \& D Team [J]. Science and Technology Management Research, 2012, 32(18):183-187.
[19] Liu Xuemei. The Measurement of Individual Tacit Knowledge: the Preliminary Development of a Simple Scale [J]. Mathematics in Practice \& Theory, 2013, 43(17):110-119.

[20] WANG Qian, LI Zuoxue, JIN Fu. Analysis of individual tacit knowledge evaluation index based on Chinese traditional thinking mode $[\mathrm{J}]$. Scientific \& Technological Progress and Policy, 2005(7):37-39. 